\title{
Effects of strain reversal on elastic and viscoelastic properties of early age cement paste beams
}

\author{
Christopher Galitz $\cdot$ Zachary Grasley
}

Received: 20 October 2014/ Accepted: 22 February 2015/Published online: 26 February 2015

(C) RILEM 2015

\begin{abstract}
Characterization of the early age properties of concrete is an ongoing area of research particularly important in understanding longer-term behavior of pre-stressed and conventional concrete members subjected to varying load histories, including strain reversals, such as may occur during placement of a concrete deck atop pre-stressed girders. Models currently in widespread use specifically exclude cases where strain reversals are present. Research presented in this paper was undertaken to refine the understanding of the viscoelastic behavior of cement paste at an early age when subject to strain reversals. A series of small-scale beams were tested in 3-point loading with the center point displaced at irregular time intervals. At some time intervals, beams were displaced negatively, inducing a strain reversal. Results indicate significant load loss in the first few days followed by steady relaxation throughout the length of testing. Results further indicate that load relaxation after reversal is significantly slower than that with samedirection increases in load. Finally, measurements of
\end{abstract}

C. Galitz

Virginia Polytechnic Institute and State University, 107 Patton Hall, 750 Drillfield Dr, Blacksburg,

VA 24061, USA

e-mail: cgalitz@vt.edu

Z. Grasley $(\bowtie)$

Texas A \& M University, 503B CE/TTI Bldg, 3136 TAMU, College Station, TX 77843, USA

e-mail: grasley@gmail.com
Young's modulus of elasticity throughout the testing program indicate that sustained loading leads to an apparent increase in beam stiffness compared to unloaded beams of the same age. The explanation and significance of these phenomena are explored.

Keywords Early age cement - Viscoelasticity · Aging $\cdot$ Young's modulus $\cdot$ Beam bending

\section{Introduction}

It has long been understood that concrete, and more specifically, the cement paste within concrete, evolves over time. Cement is a mixture of varying proportions of compounds including alite $\left(\mathrm{Ca}_{3} \mathrm{SiO}_{5}\right)$, belite $\left(\mathrm{Ca}_{2}\right.$ $\left.\mathrm{SiO}_{4}\right)$, aluminate $\left(\mathrm{Ca}_{3} \mathrm{Al}_{2} \mathrm{O}_{6}\right)$, and ferrite $\left(\mathrm{Ca}_{2} \mathrm{AlFeO}_{5}\right)$ based phases [25]. Each of these phases reacts with added water to form hydrates. Because the rate of reaction of each phase is different and the distribution of cement particle sizes is highly variable, it is difficult to predict the exact makeup of the resulting structure. However, it is generally assumed that at the macro scale (specimens $>1 \mathrm{~mm}$ in every dimension), structural properties of cement paste, both elastic and viscoelastic, are generally isotropic and homogenous and can be studied and potentially predicted.

To date, the majority of research on viscoelastic properties has been limited to macro or global-scale concrete specimens. The database for creep and 
shrinkage data used for study has resulted in four different design methods presented within applicable design practices [1]. The approach typically used within these methods is to fit equations to data while the fundamental factors causing the observed behavior are often overlooked. Further, the ACI document specifically excludes use of the methods when strain reversals are present. Though relaxation after strain reversal has been studied in several polymer materials $[11,12,19]$, including the development of complex mathematical models [10], the behavior has not to date been characterized for cementitious materials.

The purpose of the research presented herein is to broaden the understanding of the structural response of cement paste when subject to multiple load steps, with and without strain reversal, at an early age. To this end, the authors designed and performed a protocol of relaxation testing of cement paste beams subject to three-point bending. It is hoped that with further understanding of this behavior, better models can be developed that will help predict more closely the viscoelastic behavior of concrete at the global scale.

\section{Materials and methods}

Beams were cast using a cement paste mixture of ASTM C150 Type I/II Portland cement and a water:cement ratio ("w:c") of 0.40 . Batches were mixed using a paddle mixer and of sufficient size to cast $6-8$ beams in general accordance with ASTM C305-14. Exceptions to the standard were: the paddle blade used was plastic rather than stainless steel, the bowl scraper was integrated onto the outer edges of the paddle for continuous scraping, and the mixing times at slow and high speed were 1 and 5 min, respectively, with no break in between to scrape down the bowl. Beams were cast by inducing suction at one end of a square cross-section acrylic tube, nominally $15 \mathrm{~mm}$ on a side and $300 \mathrm{~mm}$ long. Ends were sealed immediately after filling and the beams cured at $21 \pm 2{ }^{\circ} \mathrm{C}$, placed horizontally on a series of rotating rods to limit segregation due to bleed water migration. After approximately $24 \mathrm{~h}$, the molds were removed and the paste beams sealed using foil-backed tape, similar to previous research [15], with seams sealed with wax. Beams were stored at the same curing temperature until and throughout loading.
A review of ASTM beam testing standards revealed that for cementitious materials, standard beams are typically of larger dimensions and loaded using thirdpoint loading. Because for the current research, the failure load was not of primary concern and in order to simplify calculation and measurement of maximum deflection, a 3-point loading was chosen for relaxation testing. The designed testing frame, as shown in Fig. 1, was constructed of $6.35 \mathrm{~mm}$ thick steel angles and plate, bolted together rigidly to provide moment connections between members, and steel rod supports placed to give a span length of approximately $250 \mathrm{~mm}$. Displacements were applied at midspan through a $12 \mathrm{~mm}^{2}$ bearing plate attached to a spherical bearing resting on the load cell below. The load cell was displaced from beneath using a lever attached to the reaction frame.

Displacements were induced by manually tightening a screw at the opposite end of the lever, holding the position in place between loadings. Where strain reversal was necessary, the beam was first unloaded and then flipped upside down for the next cycle. Midspan displacements, relative to the steel angle above, were measured at the top surface of the beam using a digital dial gage during the first $70 \%$ of load application to give sufficient data to compute the (quasi) instantaneous Young's modulus of elasticity, and the dial gage removed for the duration of the cycle. The rate at which the screw was rotated was kept relatively constant and, assuming linear elastic beam behavior, corresponded to a loading rate for each application of approximately $20 \mathrm{~N} / \mathrm{min}$ corresponding to a strain rate of approximately $0.018 \%$ per minute. The approximate displacement history for the different combinations of load cycles can be seen in Fig. 2. For reference, a displacement of $0.25 \mathrm{~mm}$ equates to a strain of approximately $0.011 \%$. The duration of each load cycle for each beam, including the initial load application, appears in Table 1 . Note that although displacement was typically not measured throughout a load cycle, the assumption that the beam midpoint does not displace during the relaxation test was verified by measurements of select beams. Further note that the testing frame rigidity, along with the use of a relative measurement between beam and frame, limited the influence of frame deflection on recorded displacements to that caused by the force of the dial gage itself, estimated to be less than $0.005 \%$ of the beam deflection. 


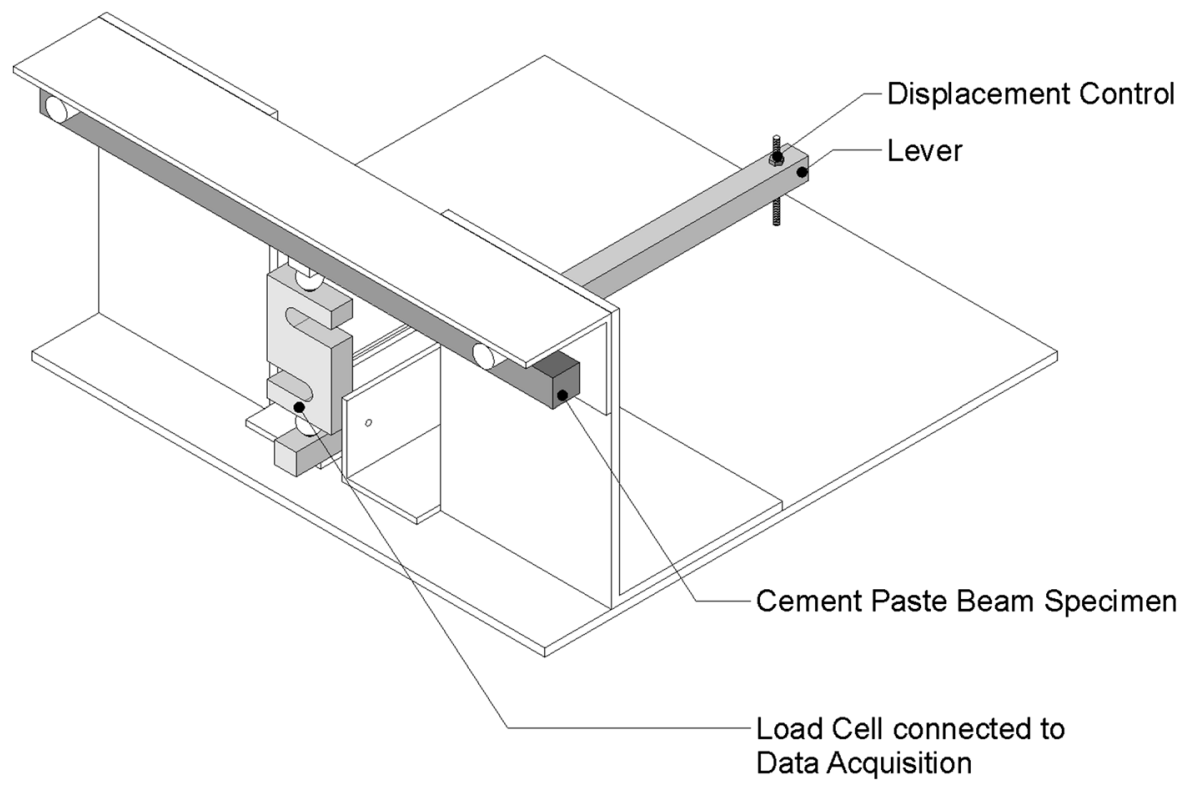

Fig. 1 Three-point beam bending test set up

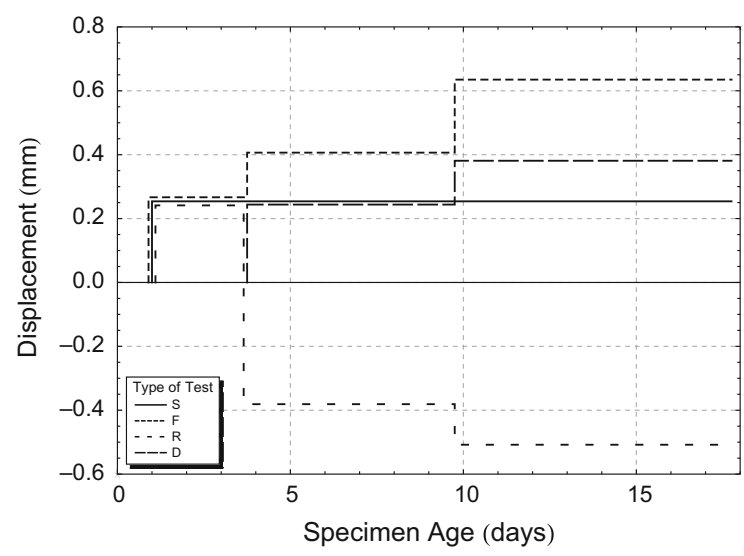

Fig. 2 Approximate stepped displacement histories defining four cement paste beam loading profiles

\section{Time-dependence of the elastic Young's modulus}

During load application for the long-term tests, displacement and load history were collected and the Young's modulus of elasticity during that loading calculated. Additionally, companion beams were cast and tested to failure for comparison purposes. A typical load to failure curve appears in Fig. 3. Note the early variations in the load-displacement relationship, likely due to initial setting of the test frame. These data were generally discarded. The superimposed line is fit to a selected portion of the data, assumed to be linear. The slight variations from the line, seen most significantly at a displacement of approximately $0.06 \mathrm{~mm}$, correspond to either slight movement of the loading screw within its seat in the lever or slight hesitations in the movement of the lever within its brackets due to friction. Both of these effects were only present when the screw was being turned and no similar variations were noticed in relaxation data. Note that the very good linearity (the coefficient of determination, $\left.R^{2}=0.951\right)$ of the lower portion of the curve $(<85 \%$ ultimate) implies that both the tensile and compressive load-displacement relationships are likely linear. It should be further noted that all beam relaxation testing described above was conducted within this lower, linear region.

By taking the slope of the load-displacement curve and assuming that the beam response may be approximated as an Euler-Bernoulli beam response, the Young's modulus of elasticity at the time of loading was determined. The results are presented in Fig. 4 with curves fit to the relationship [20]

$E=0.96 E_{\infty}\left\{1-\exp \left\{-\left[a(t-b)^{\mathrm{c}}\right]\right\}\right\}$,

where 0.96 corresponds to the maximum ratio of solids to total volume at time $t=\infty$, based on the w:c of 0.4 [23], $E_{\infty}$ is the Young's modulus of hardened material 
Table 1 Incremental load values, $\mathrm{d} P$, and cement paste age at application, $t^{\prime}$

\begin{tabular}{|c|c|c|c|c|c|c|c|}
\hline \multirow[t]{2}{*}{ Beam $^{a}$} & \multicolumn{2}{|l|}{ Cycle 1} & \multicolumn{2}{|c|}{ Cycle 2} & \multicolumn{2}{|c|}{ Cycle 3} & \multirow{2}{*}{$\begin{array}{l}\text { End } \\
t\end{array}$} \\
\hline & $t^{\prime}$ (days) & $\mathrm{d} P(N)$ & $t^{\prime}$ & $\mathrm{d} P^{\mathrm{b}}$ & $t^{\prime}$ & $\mathrm{d} P^{\mathrm{a}}$ & \\
\hline $\mathrm{C} 1 \mathrm{~S}$ & 1.16 & 29.9 & & & & & 17.00 \\
\hline $\mathrm{C} 2 \mathrm{~S}$ & 0.96 & 41.0 & & & & & 3.70 \\
\hline $\mathrm{C} 2 \mathrm{~F}$ & 0.94 & 38.7 & 3.72 & 34.1 & 9.75 & 23.4 & 17.77 \\
\hline $\mathrm{C} 2 \mathrm{R}$ & 0.95 & 41.3 & 3.72 & -61.8 & 9.75 & -28.0 & 17.76 \\
\hline $\mathrm{C} 3 \mathrm{~F}$ & 0.98 & 38.5 & 3.72 & 28.8 & 9.76 & 20.7 & 17.71 \\
\hline $\mathrm{C} 3 \mathrm{R}$ & 0.97 & 39.8 & 3.75 & -53.6 & 9.75 & -31.7 & 17.72 \\
\hline C3D1 & & & 3.75 & 44.2 & 9.75 & 35.6 & 17.73 \\
\hline C3D2 & & & 3.76 & 53.3 & 9.75 & 24.5 & 17.73 \\
\hline $\mathrm{C} 4 \mathrm{~F}$ & 1.04 & 41.8 & 3.73 & 31.1 & 9.84 & 25.6 & 17.76 \\
\hline C4R & 1.05 & 39.8 & 3.74 & -60.8 & 9.84 & -34.0 & 17.77 \\
\hline C4D & & & 3.76 & 43.9 & 9.84 & 27.4 & 17.76 \\
\hline C8S1 & 1.02 & 45.1 & & & & & 54.91 \\
\hline C8S2 & 1.02 & 44.7 & & & & & 54.91 \\
\hline $\mathrm{C} 8 \mathrm{~S} 3$ & 1.02 & 45.8 & & & & & 54.92 \\
\hline
\end{tabular}

${ }^{a}$ Beam designations $\mathrm{C} m X n$ indicate cement paste batch $\mathrm{m}$ subject to displacement history $X$ and specimen number $n$ within each batch and history combination. Histories are as depicted in Fig. 2 and can be termed "Single sustained load", "Forward loading in all cycles", "Reverse loading in second cycle" and "Delayed first loading"

b Negative load values indicate load direction opposite from original beam configuration (first loading cycle)

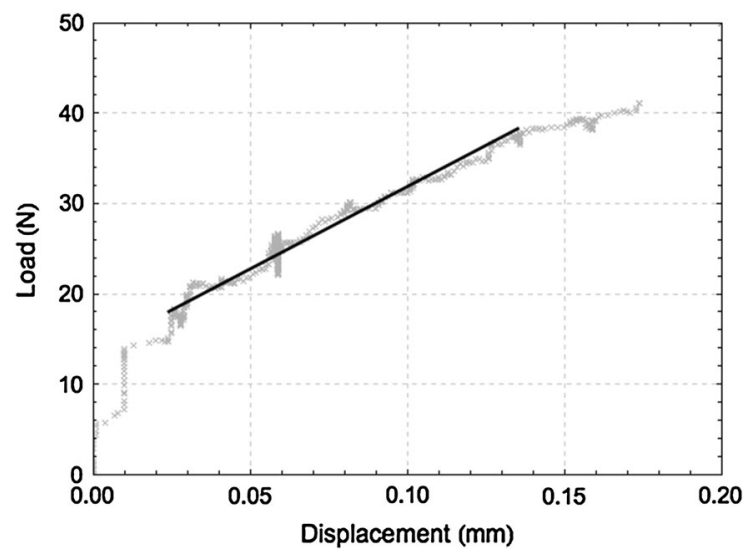

Fig. 3 Typical load-displacement to failure for cement paste beams with linear portion, after initial seating, used to determine Young's modulus of elasticity

at infinite time, $b$ represents a time delay before stiffening of the paste occurs, and $a$ and $c$ are fit parameters. The $R^{2}$ values for the first loadings and previously loaded curves are 0.988 and 0.995 , respectively. Note that the data for previously loaded specimens includes those in both the forward and

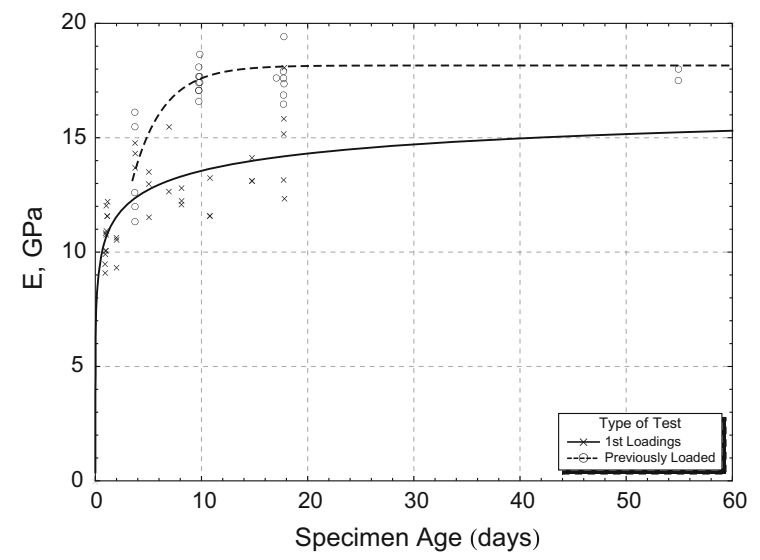

Fig. 4 Young's modulus of elasticity versus age indicating a significantly higher stiffness in cement paste beams previously loaded

reverse load direction. Close examination of individual results reveals no trend indicating that reversal of load affects the value of Young's modulus.

Based on the curve fits shown in Fig. 4, the eventual extrapolated Young's modulus of elasticity, $E_{\infty}$, is approximately $17.5 \mathrm{GPa}$ for virgin specimens and 
approximately $18.9 \mathrm{GPa}$ for specimens previously loaded.

\section{Load relaxation}

Throughout each loading cycle, the load necessary to maintain displacement was captured using an automated data acquisition system. For the first loading cycles of each specimen the data for the first 12 days are plotted in Fig. 5. Note that captured load is normalized by the initial load increment to assist in later analysis. The series of data depicted in Fig. 5 are seen to be overlapping and consistent within each group of load initiation (approximately 1 and 3.75 days). Because these are the first loadings and the specimens are replicates of the same mix design, it should be expected that the only trend between specimens would relate to specimen age at the beginning of the first loading cycle.

For the second and third loading cycles, it is first approximated that relaxation, in general accordance with the Boltzmann superposition principle, can be determined as the summation of the influence of individual load applications. Thus, during subsequent load cycles, the influence of the first load increment can be subtracted from the overall data to give the net load from the second load application. The results of this subtraction are presented in Fig. 6. Note that in the second load cycle, immediately after reversal, beams loaded in the opposite direction retain a significantly higher percentage of the applied load than those loaded in the same direction as the previous cycle.

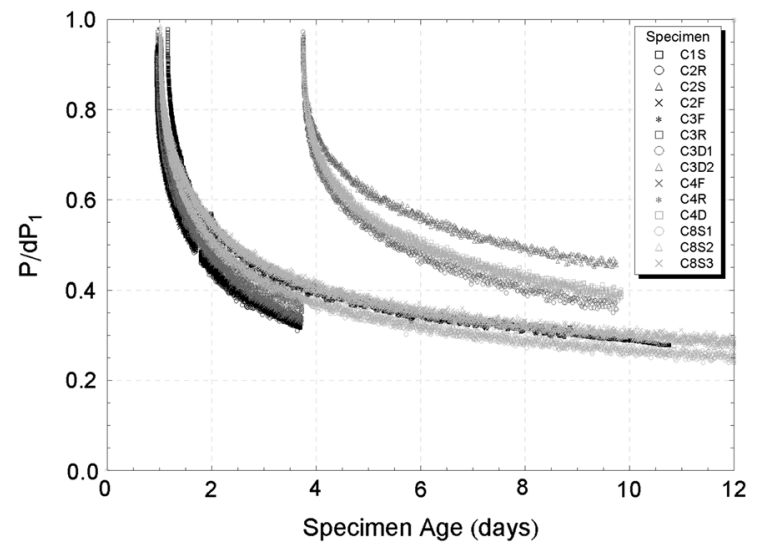

Fig. 5 Relaxation, $P / \mathrm{d} P$, during first loadings initiated at different ages

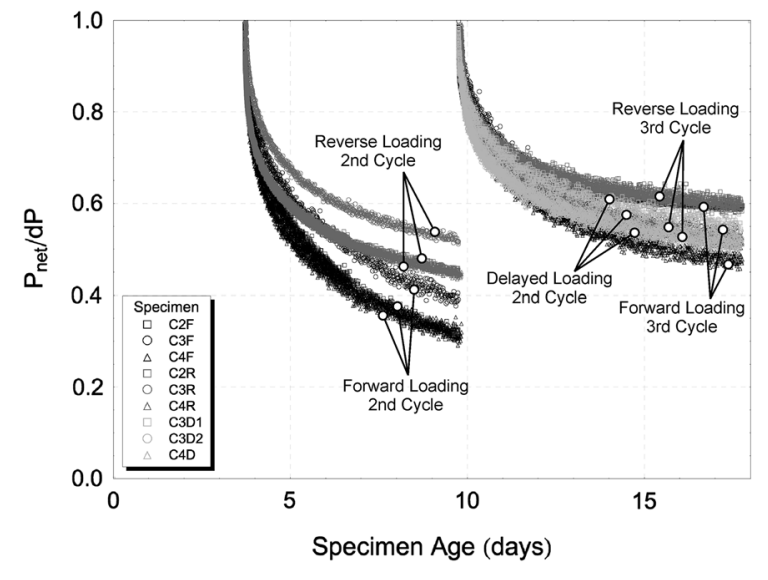

Fig. 6 Relaxation, $P_{\text {net }} / \mathrm{d} P$, during second and third loadings after the effect(s) of the previous load step(s) has been removed from the raw data, indicating significant difference in relaxation during time interval immediately following strain reversal

This trend is not as apparent in the third loading cycle. Further note the similarity in curves between the third cycle of both forward and reverse loaded specimens and the second cycle of the delayed loading specimens, all initiated at approximately 10 days.

\section{Analysis of relaxation data}

The first step in considering relaxation in the beams was to examine each portion of the data for trends and anomalies. One behavior of interest was seen in most data sets, most pronounced during the first application of load. As can be seen in Fig. 7, there is a small but

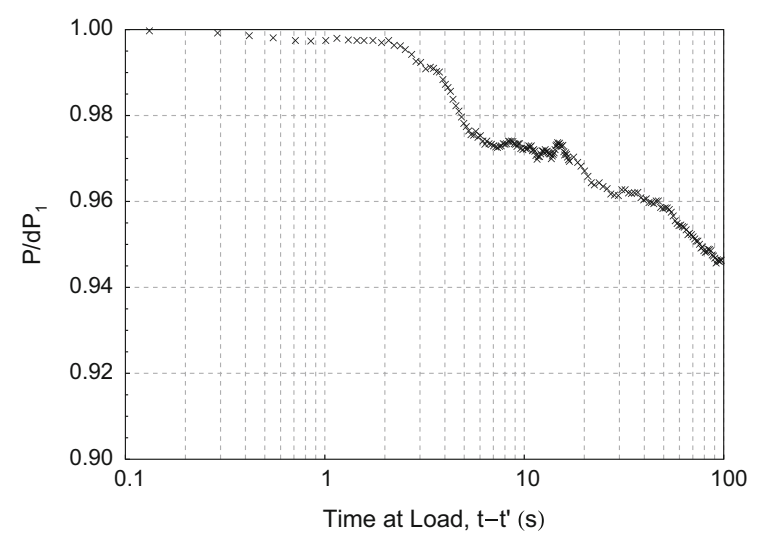

Fig. 7 Very early relaxation behavior indicating drop in load during first $10 \mathrm{~s}$ 
steep decline in retained load, typically between 1 and $10 \mathrm{~s}$, before relaxation smooths out to its long-term behavior. This phenomenon is similar to that seen in saturated beams tested by others $[26,27]$. In the cited work, the early relaxation was attributed to hydraulic movement of fluid through the pore structure of the specimen and into the surrounding fluid. In the current research, with sealed boundaries, the mechanism of this relaxation is not precisely the same. In a typical early age cement paste, even under sealed conditions, the material is not completely saturated. This allows capillary (or gel) water flow at the local level due to applied pressures [2, 14, 29], a process analogous to flow to a surrounding medium. However, as this early relaxation mechanism is not of primary interest in the current work, the first $100 \mathrm{~s}$ of data was truncated from all specimens and the associated load loss modeled as a constant, $R_{0}$, similar to the method presented by Vichit-Vadakan and Scherer, as explained in Sect. 5.2.

The second trend observed in most data sets was the tendency of the load to approach a non-zero asymptotic value. As seen in Fig. 5, the longer a particular load cycle lasted, the more pronounced the trend. This tendency was incorporated into the fit model as explained in Sect. 5.2.

With the preliminary observations, it was then possible to characterize the viscoelastic relaxation and compare to functions published elsewhere. Though several theories for modeling exist, for simplicity and ease of comparison, the solidification theory was selected for comparison to the experimental data due to its connection to actual mechanisms.

\subsection{Solidification theory}

One of the significant complications in considering the viscoelastic properties of cementitious materials is the fact that as time passes, the cement hydrates, changing its physical properties and therefore, its response to future loading. This process, called aging, has been handled in several ways in the literature, one of which is the solidification theory [3]. The theory considers aging as a solidification process such that during any change in time, additional hydration products form, with each new segment created in a stress-free state and with age-independent physical properties. As later explained [5, 9], the response to a flexural or uniaxial relaxation test such as considered currently, can be characterized by the constitutive relation $\sigma(t)=\int_{t^{\prime}=0}^{t} \Psi\left(t-t^{\prime}\right) v\left(t^{\prime}\right) \mathrm{d} \varepsilon\left(t^{\prime}\right)$,

where $\sigma(t)$ is the axial stress at time $t, v$ is the volume fraction of solidified material, $\mathrm{d} \varepsilon$ is the change in axial strain, and $\Psi$ is the uniaxial relaxation function. For a beam relaxation test whereby displacement is induced at specific times, $t^{\prime}$, the integral goes to zero at all $t$ not equal to $t^{\prime}$. Replacing $\Psi$ with $E_{\infty} \Psi^{*}$ and converting to a summation of discrete steps in strain, yields

$\sigma(t)=\sum_{i=1}^{n} E_{\infty} \Psi^{*}\left(t-t_{i}\right) v_{i} \mathrm{~d} \varepsilon_{i}$

where $n$ is the total number of displacements, each at time $t_{i}$, and $E_{\infty}$ is the Young's elastic modulus of a nonrelaxing cement paste once all hydration has occurred.

In a simply-supported, 3-point loading, prismatic beam test, assuming that the beam behaves as an EulerBernoulli beam, $\sigma$ and $\varepsilon$ can be replaced by functions of the applied load $P$ and displacement $\delta$, such that

$\frac{3 L}{2 h^{3}} P(t)=\sum_{i=1}^{n} E_{\infty} \Psi^{*}\left(t-t_{i}\right) v_{i} \frac{6 h}{L^{2}} \mathrm{~d} \delta_{i}$,

where $L$ is the length of the beam and $h$ is the height and width of a square cross-section beam. Collecting constants and moving the modulus outside the summation yields

$P(t)=\frac{4 E_{\infty} h^{4}}{L^{3}} \sum_{i=1}^{n} \Psi^{*}\left(t-t_{i}\right) v_{i} \mathrm{~d} \delta_{i}$.

Consider the case where $n=1$ (a single displacement at time $t_{1}$ with a corresponding load increase $\left.\mathrm{d} P_{1}\right)$. Knowing that at $t=t_{1}, \Psi^{*}$ must be equal to 1 , it can be seen that,

$\mathrm{d} P_{1}=\frac{4 h^{4} E_{\infty} \mathrm{d} \delta_{1} v_{1}}{L^{3}}$,

and therefore for $t>t_{1}$,

$P(t)=\mathrm{d} P_{1} \Psi^{*}\left(t-t_{1}\right)$.

Now consider the case where $n=2$. The load, $P$, just before the second displacement, is simply $\mathrm{d} P_{1 \text { - }}$ $\Psi *\left(t_{2}-t_{1}\right)$. Just after the displacement, with $t=t_{2}$,

$$
\begin{aligned}
P\left(t_{2}\right) & =\mathrm{d} P_{1} \Psi^{*}\left(t_{2}-t_{1}\right)+\mathrm{d} P_{2} \\
& =\frac{4 E_{\infty} h^{4}}{L^{3}} \Psi^{*}\left(t_{2}-t_{1}\right) v_{1} \mathrm{~d} \delta_{1}+E_{\infty} v_{2} \mathrm{~d} \delta_{2} .
\end{aligned}
$$


Recognizing that the first term on the right-hand side of Eq. 8 is the load response to the first displacement and the second term is that to the second, it can be generalized for any number of induced displacements,

$P(t)=\sum_{i=1}^{n} \mathrm{~d} P_{i} \Psi^{*}\left(t-t_{i}\right)$

The function, $P(t)$ in Eq. 9 implies that the relaxation function can be determined, independent of aging, and without consideration of the magnitudes of displacements or $E$ values. For a single load cycle, the $\Psi *$ function can be determined by normalizing the current load by the initial load, as was presented in the figures above. Because the solidification theory relies on the assumption that cement paste solidifies in a zero-stress condition, when displacement is held constant, the increased volume of solidified products has no influence on material response to previously applied loads. Thus, for a particular load step, the volume fraction is a constant and normalizing by the initial load removes this variable. Likewise, as the Young's modulus of elasticity of a particular volume fraction is a function only of time, the normalization also removes this variable, leaving only the relaxation function, $\Psi^{*}$. This same function can then be used throughout all load cycles.

As an illustration, consider the curves presented in Fig. 6. If the solidification theory holds, and assuming that the influence of earlier load cycles has been appropriately subtracted, the resulting relaxation curves for second and third cycles should be the same, merely shifted in time (horizontally). As can be seen, relaxation of load is significantly over-predicted by the solidification theory for displacement increments after the first. This implies that relaxation is retarded by the aging of the hydration products, i.e., viscoelastic aging is not fully characterized by the solidification model. This same conclusion was drawn in previous research $[7,13]$.

\subsection{Fitting the relaxation functions}

As noted above, there were two trends apparent in the data sets, an early offset and an eventual lower threshold. The first trend can be simulated by fitting the data to a function $R_{0} \Psi^{*}$ where $R_{0}$ represents the constant associated with hydraulic relaxation after some short time duration, consistent with the work of Vichit-Vadakan and Scherer [27]. For the second trend, the fit model for $\Psi^{*}$ must include a constant term. This leaves the span in between the upper and lower limits to be fit. Though several models have been used to characterize relaxation curves, the most common corresponds to a series of dashpots and spring combinations (e.g., Maxwell model). For the current case, and incorporating the modifications mentioned above, the resulting fit model for a particular load cycle can be written as

$$
\begin{aligned}
\frac{P(t)}{\mathrm{d} P} & =R_{0} \Psi^{*}\left(t-t^{\prime}\right) \\
& =R_{0}\left[\sum_{i=1}^{n} a_{i} \exp \left(-\frac{t-t^{\prime}}{\tau_{i}}\right)+b\right]
\end{aligned}
$$

where $\tau_{i}$ are times typically chosen as regular intervals during the span of interest, $b$ is the asymptotic value, and $a_{i}$ are weighting factors used as fit parameters (summed to 1 ). The value of $n$, the number of terms needed to obtain a good fit, is somewhat arbitrary. However, for purposes of this paper, seven terms were found to be sufficient, corresponding to the rheologic model of a Maxwell chain of seven parallel elements with each element consisting of a spring and dashpot in series.

Using a nonlinear fitting routine within Mathematica [28], and assigning $\tau_{i}$ at equal intervals of log time from 0.0001 to 100 days, a method in use for many years [4], fit parameters were determined for the results of each specimen, with the coefficients $a_{i}$ and $b$ shown in Table 2 . The value of $R_{0}$, the initial reduction attributed to very early hydraulic relaxation varied between 0.94 and 1.00 , corresponding to a maximum value of load loss of $6 \%$ and indicating a relatively minor effect compared to long-term relaxation. The coefficient of determination, $R^{2}$, exceeded 0.9994 for all $\Psi^{*}$ curves. An example fit for the first loading cycle is included as Fig. 8.

Consideration of data in Table 2 reveals several key points. First, the highest weighting factors are within the region of the age of the specimen at time of load, $\tau=1$ for the first cycle and $\tau=10$ for later cycles. Though the values of $\tau$ do not correspond directly to physical processes, the shift in relative weighting factors to later time intervals implies that aging of the cement has an observable effect on material response beyond that predicted by solidification. Second, the 
Table 2 Fit results for relaxation functions

\begin{tabular}{|c|c|c|c|c|c|c|c|c|}
\hline$\tau$ (days) & $\begin{array}{l}0.0001 \\
a_{1}\end{array}$ & $\begin{array}{l}0.001 \\
a_{2}\end{array}$ & $\begin{array}{l}0.01 \\
a_{3}\end{array}$ & $\begin{array}{l}0.1 \\
a_{4}\end{array}$ & $\begin{array}{l}1 \\
a_{5}\end{array}$ & $\begin{array}{l}10 \\
a_{6}\end{array}$ & $\begin{array}{l}100 \\
a_{7}\end{array}$ & $b$ \\
\hline \multicolumn{9}{|c|}{ Cycle 1 at 1 day } \\
\hline C1S & 0.071 & 0.072 & 0.064 & 0.157 & 0.311 & 0.247 & 0.077 & 0.127 \\
\hline $\mathrm{C} 2 \mathrm{~S}$ & 0.034 & 0.053 & 0.080 & 0.148 & 0.372 & 0.222 & 0.091 & 0.051 \\
\hline $\mathrm{C} 2 \mathrm{~F}$ & 0.002 & 0.028 & 0.084 & 0.151 & 0.385 & 0.000 & 0.349 & 0.000 \\
\hline $\mathrm{C} 2 \mathrm{R}$ & 0.015 & 0.034 & 0.092 & 0.142 & 0.394 & 0.015 & 0.308 & 0.010 \\
\hline $\mathrm{C} 3 \mathrm{~F}$ & 0.001 & 0.070 & 0.075 & 0.107 & 0.415 & 0.041 & 0.292 & 0.063 \\
\hline $\mathrm{C} 3 \mathrm{R}$ & 0.044 & 0.169 & 0.069 & 0.150 & 0.348 & 0.049 & 0.171 & 0.154 \\
\hline $\mathrm{C} 4 \mathrm{~F}$ & 0.061 & 0.081 & 0.099 & 0.134 & 0.376 & 0.118 & 0.131 & 0.111 \\
\hline $\mathrm{C} 4 \mathrm{R}$ & 0.000 & 0.036 & 0.082 & 0.133 & 0.373 & 0.014 & 0.362 & 0.000 \\
\hline $\mathrm{C} 8 \mathrm{~S} 1$ & 0.063 & 0.063 & 0.072 & 0.137 & 0.328 & 0.245 & 0.093 & 0.092 \\
\hline $\mathrm{C} 8 \mathrm{~S} 2$ & 0.038 & 0.058 & 0.069 & 0.117 & 0.346 & 0.234 & 0.137 & 0.088 \\
\hline $\mathrm{C} 8 \mathrm{~S} 3$ & 0.061 & 0.050 & 0.067 & 0.126 & 0.329 & 0.250 & 0.118 & 0.101 \\
\hline \multicolumn{9}{|c|}{ Cycle 2 at 4 days } \\
\hline $\mathrm{C} 2 \mathrm{~F}$ & 0.007 & 0.012 & 0.052 & 0.130 & 0.256 & 0.529 & 0.013 & 0.013 \\
\hline $\mathrm{C} 2 \mathrm{R}$ & 0.023 & 0.044 & 0.074 & 0.126 & 0.203 & 0.231 & 0.298 & 0.061 \\
\hline $\mathrm{C} 3 \mathrm{~F}$ & 0.043 & 0.035 & 0.048 & 0.107 & 0.202 & 0.532 & 0.033 & 0.075 \\
\hline C3R & 0.000 & 0.000 & 0.037 & 0.115 & 0.209 & 0.230 & 0.409 & 0.009 \\
\hline C3D1 & 0.064 & 0.050 & 0.097 & 0.144 & 0.204 & 0.361 & 0.079 & 0.103 \\
\hline C3D2 & 0.072 & 0.059 & 0.077 & 0.109 & 0.176 & 0.329 & 0.178 & 0.121 \\
\hline $\mathrm{C} 4 \mathrm{~F}$ & 0.000 & 0.000 & 0.095 & 0.150 & 0.249 & 0.407 & 0.098 & 0.000 \\
\hline $\mathrm{C} 4 \mathrm{R}$ & 0.025 & 0.045 & 0.068 & 0.136 & 0.207 & 0.248 & 0.272 & 0.064 \\
\hline C4D & 0.071 & 0.070 & 0.080 & 0.139 & 0.215 & 0.338 & 0.085 & 0.136 \\
\hline \multicolumn{9}{|c|}{ Cycle 3 at 10 days } \\
\hline $\mathrm{C} 2 \mathrm{~F}$ & 0.018 & 0.066 & 0.056 & 0.062 & 0.175 & 0.058 & 0.565 & 0.054 \\
\hline $\mathrm{C} 2 \mathrm{R}$ & 0.119 & 0.038 & 0.021 & 0.088 & 0.171 & 0.114 & 0.449 & 0.148 \\
\hline $\mathrm{C} 3 \mathrm{~F}$ & 0.316 & 0.000 & 0.046 & 0.054 & 0.207 & 0.377 & 0.000 & 0.375 \\
\hline C3R & 0.001 & 0.034 & 0.040 & 0.085 & 0.192 & 0.217 & 0.431 & 0.041 \\
\hline C3D1 & 0.087 & 0.021 & 0.034 & 0.083 & 0.189 & 0.192 & 0.393 & 0.119 \\
\hline $\mathrm{C} 3 \mathrm{D} 2$ & 0.038 & 0.044 & 0.032 & 0.121 & 0.197 & 0.152 & 0.417 & 0.088 \\
\hline $\mathrm{C} 4 \mathrm{~F}$ & 0.055 & 0.065 & 0.042 & 0.097 & 0.229 & 0.263 & 0.249 & 0.136 \\
\hline C4R & 0.051 & 0.061 & 0.043 & 0.087 & 0.199 & 0.235 & 0.325 & 0.112 \\
\hline C4D & 0.129 & 0.058 & 0.036 & 0.058 & 0.173 & 0.187 & 0.358 & 0.109 \\
\hline
\end{tabular}

eventual value of the relaxation function, signified by $b$, varies significantly. For the load cycles of longest duration, specifically batch $\mathrm{C} 8$, the values are similar and non-zero, verifying the suspected asymptote of the fit curve. Considering that each term of the fit is reduced to essentially zero within an order of magnitude of time after $\tau$, this asymptote would be reached at a cement paste age less than 1000 days. Lastly, the high variability in the weighting coefficients from specimen to specimen and batch to batch, even within the same loading cycle and load directions, indicates that as a general rule, a simpler model may be more appropriate. The extra terms used here can more accurately capture small variations in the data where a simpler model may more readily be modified for additional parameters such as temperature or w:c ratio while retaining sufficient accuracy of the fit.

To better illustrate the consistency of the data for the first load cycle, results for all specimens loaded for the first time at approximately 1 day and those at 4 days were combined into two single data sets and fits generated. Figure 9 shows the fit curve with the 


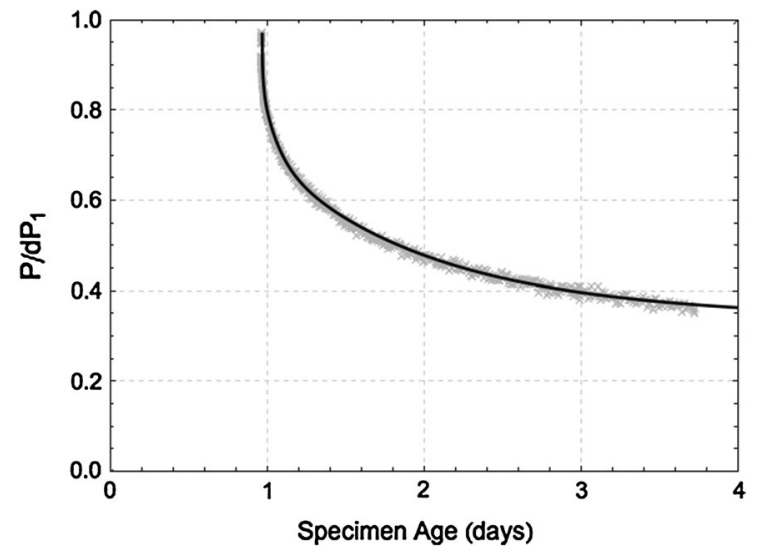

Fig. 8 Example of curve fit to raw relaxation data for first loading cycle of specimen

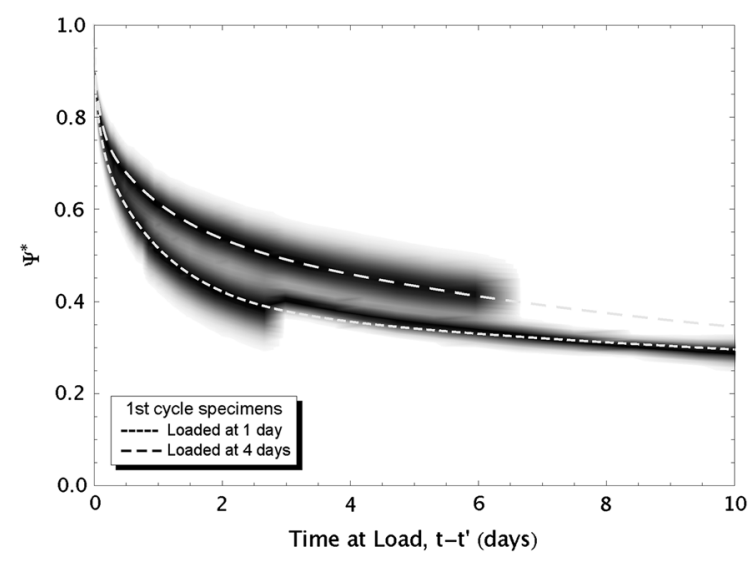

Fig. 9 Probability density of first loading cycle data compared to derived fit curves showing a significant difference in relaxation between samples first loaded at 1 day and those first loaded at 4 days

probability density distribution of the data represented as shaded regions on each side of the curve. Note that the age at first loading has a significant influence on the relaxation function throughout early age but the curves tend to converge as the time at load exceeds 10 days. Were the graph extended to the full range of the test ( $>53$ days), it would be seen that loads on the younger specimens relax to an asymptote of approximately $14.5 \%$ of the original load while the older specimens approach $1.2 \%$ at infinite time. Given the anomalies in the fit for certain portions of the curve, these eventual values certainly have associated error. However, through the time span of the tests currently under consideration, the density of the data depicted in the figure shows that use of a single fit curve to represent multiple specimens loaded at similar ages is appropriate.

\subsection{Effect of strain reversal}

Given the results of fits described in the previous section, data presented in Fig. 6 was similarly grouped for comparison purposes. For each second loading cycle, the projected effects of the first loading on the same specimen, using curve fits previously generated, were subtracted from the data. The results, after dividing out the $R_{0}$ term for the second cycle, are presented in Fig. 10. Note that data is limited to specimens with a first loading cycle at 1 day.

As seen in the figure, at later ages (time at load $>5$ days), there is virtually no overlap of probability density of the two curves and thus, virtually no statistical likelihood that the relaxation function for specimens subject to strain reversal is the same as that for forward-loaded specimens. The load eventually relaxes to approximately $0.35 \%$ of original for forward-loaded specimens (i.e., the second step load was of the same sign as the first) while for reverse specimens (i.e., the second step load was opposite in sign to the first) the eventual relaxed load is $14.8 \%$ of the initial. This gap between the curves develops over the first few days, reaching $14 \%$ at the end of 6 days of the second loading cycle (10 days of total aging).

Considering the variability inherent in comparing specimens from different batches, and to compare stress effects directly, the relaxation data was analyzed

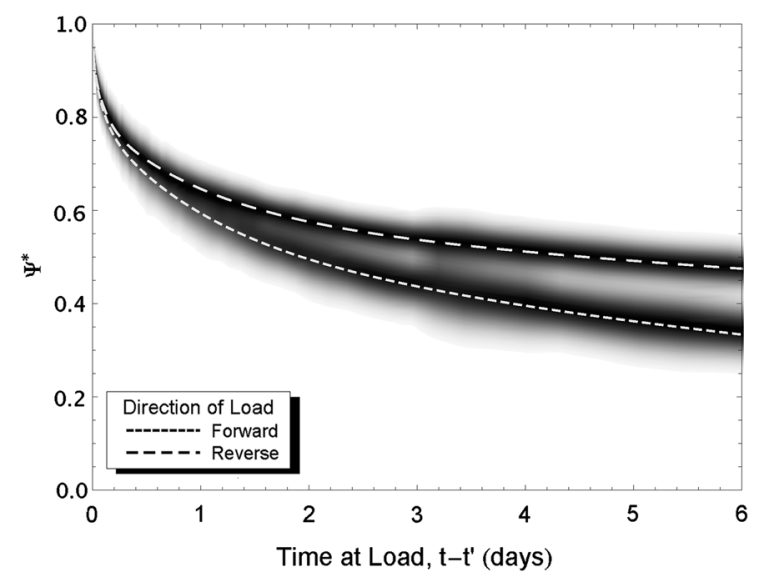

Fig. 10 Probability density of second loading cycle data with derived fit curves comparing relaxation in beams subject to strain reversal to those with same direction load steps 


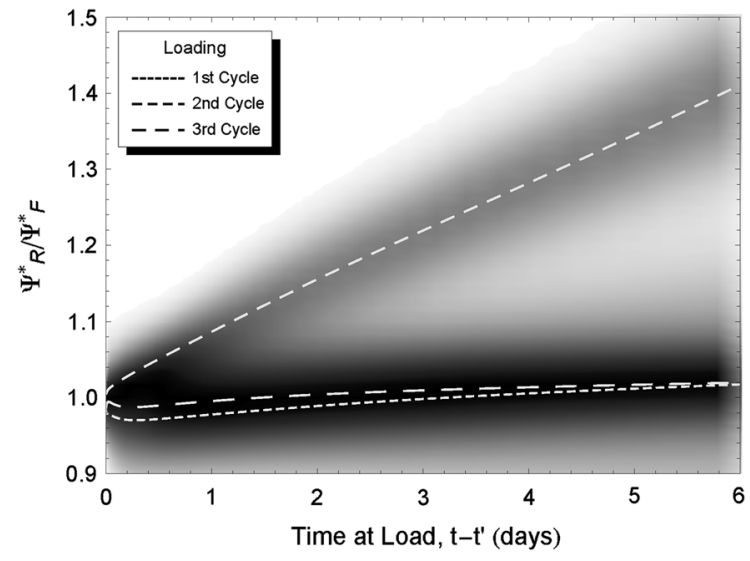

Fig. 11 Ratio of relaxation, reverse to forward loaded specimens during each of three loading cycles indicating the significant influence of strain reversal on relaxation behavior

in an additional manner. For three batches, there were companion specimens loaded in the forward and reverse directions in loading cycles 2 and 3. By reducing the data to the $\Psi^{*}$ fit curves only and dividing the reverse curves by the forward curves for each cycle, Fig. 11 was generated, taking the average for each grouping of three specimens (three different batches). This ratio compares curves of equal age during each cycle, eliminating the viscoelastic aging effects seen in previous figures. Note that the relaxation function for later cycles is based on the net load for that individual load step, meaning the effect of earlier cycles has been subtracted out using the Boltzmann superposition theory. Further note that in the first cycle, the load direction for companion specimens is the same and the ratio is theoretically 1.0.

Examination of Fig. 11 reveals clearly that load and strain reversal have a significant effect throughout the life of the specimen whereas relaxation due to forward loading at later cycles is virtually indistinguishable from the relaxation during the very first load cycle. It is also interesting to note the curvature of the first and third load cycles. If there were no aging effects present other than those accounted for by the solidification theory, the curves would be flat lines at $\Psi^{*}=1.0$.

\section{Discussion}

Taken individually, results presented above for elastic Young's modulus and relaxation might be explained by previously published theories. Starting with relaxation, it is apparent that intrinsic viscoelastic aging must be accounted for in order to produce higher precision models. However, by limiting comparisons to specimens of similar age, this issue can be set aside. That leaves the readily seen difference in relaxation behavior between specimens loaded at multiple steps in a single direction versus those with a strain reversal in the loading history.

At first glance, the reduction in relaxation rate (higher retention of load) in reversed specimens lines up with the theory of relaxation being a function, at least in part, of microcracking in the structure [6, 8]. A forward load causes cracking primarily in the tension regions of the beam to grow under additional load. Essentially, relaxation compounds with same direction loading. This effect is somewhat offset by aging, as seen in comparing Figs. 9 and 10 where a higher load retention is present, even after multiple loads are applied. When a beam is reversed (or strain is reversed), the tension face goes into compression, tending to close the pre-existing cracks and starting new crack patterns in the face previously under compression.

Similarly, the trends seen in the elastic Young's modulus of loaded specimens is consistent with existing theories and previously published research $[17,18]$. The shapes of the two curves in Fig. 4 suggest that loaded specimens develop stiffness more quickly than previously unloaded specimens. As stiffness is typically a direct function of the degree of hydration, this suggests that load applied to a specimen accelerates hydration, as has been reported previously [24], possibly due to stress-induced dissolution of the cement grains [16].

When taken together, however, trends are less intuitive. The microcracking that might explain the relaxation results would, in theory, also reduce stiffness during loading. As stated above, the results for Young's modulus of elasticity of forward versus reverse specimens are indistinguishable. This apparent contradiction implies that a mechanism other than or in addition to microcracking is responsible for relaxation behavior.

A full consideration of other mechanisms potentially responsible for observed behavior is beyond the scope of this work. However, there are certain aspects of the current testing that may provide some additional insight. In Sect. 5.2, it was noted that the early 
relaxation observed could be attributed to movement of pore fluid within the specimen. For later analysis, the presence and movement of this pore fluid was ignored, consistent with the assumptions of the solidification model that only hydrated cement paste carries load. If this limiting assumption is removed, and the pressure exerted on and by the pore fluid is considered, relaxation behavior may be more fully understood. In the quasi-instantaneous loading stage, if the loads are carried by both the pore fluid and the solid structure, the fluid would be pressurized in compressive regions of the specimen and tend to flow into adjoining empty pores within that structure. This flow would be hampered by the small size of the capillaries present in the material. After some time, the fluid would equalize at a local level, relieving pressure and therefore, relieving load at the macro scale. The magnitude of load and the time required to reach equalization would be a function of both the initial proportion of water, as determined by the w:c ratio, and by the rate at which capillaries shrink, related to the degree of hydration. The general shape of the load relaxation curve due to this fluid movement would resemble that seen in Fig. 5. Determining the magnitude and precise shape of such a relaxation curve would require testing and analysis beyond the scope of this paper.

Considering the fluid movement described above in combination with the hydration process described by the solidification model can at least partially explain the time-dependent behavior seen in the testing results. At the time of initial loading, the specimen is composed of a mixture of hydrated solids, pore fluid, and air. The proportions of each of these components is related to the original w:c ratio and the timedependent degree of hydration. At earlier ages of initial loading, the relative proportion of pore fluid is higher and the capillary sizes within the hydration products larger, as compared to later ages. At later ages, with less pore fluid and more empty voids, the amount of load carried by the fluid would be less and therefore the total potential for load relaxation due to fluid migration also less. This difference in pore fluid volume available to carry load would lead to less overall early load relaxation in specimens loaded at later ages. Furthermore, the rate of relaxation is influenced by the dissolution of load bearing cement grains, which decreases at later ages thus decreasing relaxation rate $[21,22]$.

\section{Conclusions}

Sustained load alters the manner in which cement paste, and therefore concrete, reacts to additional load. This change is typically not accounted for in concrete design practice and may in some cases provide additional capacity while in others lead to unforeseen complications.

Strain reversal in a loading history results in a significantly lower rate of relaxation than would otherwise be predicted. This longer retention of applied loads could lead to unpredictable long-term performance.

The solidification theory is insufficient to fully explain viscoelastic aging. Significant differences in the relaxation rates between specimens loaded at 1 day and those loaded at 4 days show that aging functions in addition to or in lieu of solidification based aging should be incorporated into any prediction model. Such functions previously published may not be appropriate for use with cement paste analysis.

Measuring the instantaneous Young's modulus of elasticity of virgin specimens may not be sufficient to predict global scale structural performance. The presence of sustained load, regardless of sign or direction, leads to an apparent increase in stiffness of cement paste beams. This increase, as much as $10 \%$ in the long term, can have a significant effect on load distributions and deformations of large-scale structures.

The results of relaxation testing presented herein are far from inclusive of all stress states likely to be encountered in typical early-age structures. Additional testing, including uniaxial and bending, should be conducted to better characterize behavior.

Although relaxation and creep are generally considered to be based on similar mechanisms within cement paste, it is quite probable that factors such as dissolution and microcracking do not directly correlate in the two styles of test. Therefore, applying similar load histories in a creep configuration may help define which mechanisms are most responsible for observed behavior.

It is suspected that the macro-scale behavior seen here is highly dependent on stress-induced changes at the micro-scale. Therefore, additional investigation at the micro-scale, both through testing and theoretical modeling, should be performed.

The testing conducted as part of this research was limited in scope to a single water:cement ratio and a 
sealed condition to limit the influence of drying shrinkage and drying creep. These phenomena may have significant effect on the results and thus, the scope of testing should be broadened to consider the relative influence of these and other factors.

\section{Practical implications}

A typical pre-stressed beam or girder starts as pretensioned tendons surrounded by freshly placed concrete. After approximately 1 day, the tendons are released from their external anchorage and the beam is subject to a compressive force and negative moment, in addition to carrying its own weight once upward displacement occurs. After some period of time, the beam is placed on its permanent supports in the field and subject to additional dead and live loads, imparting positive moments. Though a beam in this configuration is rarely held to a constant displacement or subject to a constant load, the basic results of the current research do apply to its behavior. It should be expected that behavior of the beam after the strain reversal induced upon placement in service is not the same as that predicted based on single load-direction laboratory tests. Thus, a single model for creep and/or relaxation does not apply to all steps in the beam's actual load history. Thus, standard design practice, which tends to use a single model for loads before and after strain reversal, may not accurately predict actual beam behavior.

Acknowledgments This material is based upon work supported by the National Science Foundation under Grant No. 1327314

\section{References}

1. ACI (2008) ACI 209.2R-08 guide for modeling and calculating shrinkage and creep in hardened concrete. American Concrete Institute, Farmington Hills

2. Acker P (2004) Swelling, shrinkage and creep: a mechanical approach to cement hydration. Mater Struct 37:237-243

3. Bazant ZP (1977) Viscoelasticity of solidifying porous material—concrete. J Eng Mech 103:1049-1067

4. Bazant ZP, Asghari A (1974) Computation of age-dependent relaxation spectra. Cem Concr Res 4:567-579. doi:10. 1016/0008-8846(74)90007-6

5. Bazant ZP, Prasannan S (1989) Solidification theory for concrete creep I. Formulation. J Eng Mech 115:1691-1703

6. Bazant ZP, Zubelewicz A (1988) Strain-softening bar and beam: exact non-local solution. Int $\mathrm{J}$ Solids Struct 24:659-673. doi:10.1016/0020-7683(88)90015-7
7. Bazant ZP, Hauggaard AB, Baweja S, Ulm F-J (1997) Microprestress-solidification theory for concrete creep. I: aging and drying effects. J Eng Mech 123:1188-1194

8. Bernard O, Ulm F-J, Germaine JT (2003) Volume and deviator creep of calcium-leached cement-based materials. Cem Concr Res 33:1127-1136

9. Carol I, Bazant ZP (1993) Viscoelasticity with aging caused by solidification of nonaging constituent. J Eng Mech 119:2252-2259

10. Drozdov AD (1998) Modelling an anomalous stress relaxation in glassy polymers (the Kitagawa effect). Math Comput Model 27:45-67. doi:10.1016/S0895-7177(98)00072-7

11. Drozdov AD (2010) Time-dependent response of polypropylene after strain reversal. Int J Solids Struct 47:3221-3233. doi:10.1016/j.ijsolstr.2010.08.001

12. Gramespacher H, Meissner J (1995) Reversal of recovery direction during creep recovery of polymer blends. J Rheol 39:151-160. doi:10.1122/1.550696

13. Grasley ZC, Lange DA (2007) Constitutive modeling of the aging viscoelastic properties of Portland cement paste. Mech Time Depend Mater 11:175-198

14. Grasley ZC, Leung CK (2011) Quasi-static axial damping of poroviscoelastic cylinders. J Eng Mech 137:561-570

15. Grasley Z, Lange D, D’Ambrosia M (2006) Internal relative humidity and drying stress gradients in concrete. Mater Struct 39:901-909. doi:10.1617/s11527-006-9090-3

16. Grasley ZC, Rajagopal K, Li X (2015) Theoretical basis for dissolution-induced viscoelastic and viscoplastic behavior of cementitious materials. Cem Concr Compos (in press)

17. Harsh S, Shen Z, Darwin D (1990) Strain-rate sensitive behavior of cement paste and mortar in compression. ACI Mater J 87:508-516

18. Inozemtsev UP (1995) Cement paste and concrete hardening under strain: I. Cement paste research. Cem Concr Res 25:231-239

19. Jansons J, Aniskevich A, Pazhe L (2012) Analysis of reversible and irreversible strains in the creep of a nonlinear viscoelastic polymer. Mech Compos Mater 48:209-216. doi:10.1007/s11029-012-9266-6

20. Jiang L, Zhang Y, Hu C, Li Z (2012) Calculation of elastic modulus of early-age cement paste. Adv Cem Res 24:193-201

21. Li X, Grasley Z, Bullard J, Garboczi E (2015) Computing the time evolution of the apparent viscoelastic/viscoplastic Poisson's ratio of hydrating cement paste. Cem Concr Compos 56:121-133

22. Li X, Grasley ZC, Garboczi EJ, Bullard JW (2015) Modeling the apparent and intrinsic viscoelastic relaxation of hydrating cement paste. Cem Concr Compos 55:322-330. doi:10.1016/j.cemconcomp.2014.09.012

23. Powers TC, Brownyard TL (1946) Studies of physical properties of hardened Portland cement paste. Am Concr Inst J 18(8):934-969

24. Tamtsia BT, Beaudoin JJ, Marchand J (2004) The early age short-term creep of hardening cement paste: load-induced hydration effects. Cem Concr Compos 26:481-489

25. Taylor HFW (1990) Cement chemistry. Academic Press, San Diego

26. Valenza JJ, Thomas JJ (2012) Permeability and elastic modulus of cement paste as a function of curing temperature. Cem Concr Res 42:440-446 
27. Vichit-Vadakan W, Scherer GW (2003) Measuring permeability and stress relaxation of young cement paste by beam bending. Cem Concr Res 33:1925-1932

28. Wolfram (2012) Mathematica, Version 9.0 edn. Wolfram, Champaign
29. Wyrzykowski M, Lura P (2014) The effect of external load on internal relative humidity in concrete. Cem Concr Res 65:58-63. doi:10.1016/j.cemconres.2014.07.011 